\title{
THEORETICAL ASSESSMENT OF THE FULL-MOMENT-TENSOR RESOLVABILITY FOR RECEIVER ARRAYS USED IN MICROSEISMIC MONITORING
}

\author{
František STANĚK ${ }^{1,2,3)}$, Leo EISNER ${ }^{3,4)}$ and Aldo VESNAVER ${ }^{1)}$ \\ 1) The Petroleum Institute, Dept. of Geosciences, P.O. Box 2533, Abu Dhabi, United Arab Emirates \\ ${ }^{2)}$ Charles University in Prague, Faculty of Mathematics and Physics, Dept. of Geophysics, V Holešovickách 2, \\ 18000 Praha 8, Czech Republic \\ 3) Czech Academy of Sciences, Institute of Rock Structure and Mechanics, Dept. of Seismotectonics, V Holešovičkách 41 , \\ 18209 Praha 8, Czech Republic \\ 4) Seismik s.r.o., Kubišova 1265/8, 18200 Praha 8, Czech Republic \\ *Corresponding author's e-mail: StanekFr@irsm.cas.cz
}

\begin{tabular}{l} 
ARTICLE INFO \\
\hline Article history: \\
Received 8 September 2016 \\
Accepted 13 February 2017 \\
Available online 29 March 2017 \\
\hline
\end{tabular}

Keywords:

Microseismic monitoring

Source mechanism

Moment tensor

Inversion

Receiver array

Condition number

Reservoir

\begin{abstract}
An inversion of full moment tensors representing source mechanisms is becoming routinely used when interpreting microseismic monitoring. However, a stability of such inversion varies significantly for different receiver arrays. Unlike stability of location, which is hard to assess, the stability of inverted full moment tensor can be measured by a condition number. We tested three types of receiver arrays (dense surface array, dual borehole array and multi-borehole array) used in the microseismic monitoring and computed the theoretical stability of full moment tensor inversion from P- or P- and S-wave amplitudes at areas of interest. We analyzed a 3D distributions of the condition numbers and show that with surface and multi-borehole arrays of receivers we are capable of inverting stable source mechanisms for the events located at depths comparable to the maximum offset of receivers, but shallower events and the events at the depths of the borehole arrays are poorly constrained. This study provides a guidance for a quick prediction of source mechanism inversion quality. Computing condition numbers does not require any information about the data, it measures a theoretical quality of the moment tensor inversion. The computed condition numbers represent inversion for ideal dataset with the selected geometry.
\end{abstract}

\section{INTRODUCTION}

Microseismic monitoring is used to evaluate an efficiency of reservoir stimulation with the goal of improving production from low permeability formations. Source mechanisms are retrieved along with microseismic event locations mapping the geometry of the hydraulic fracture. These mechanisms provide an additional information on the activated fault or fracture orientation, the direction of the slip relative to the fault plane and may also resolve if microseismic events have a shear or a (partly) nonshear component. Such additional information is used to determine geomechanical parameters of the stimulated rock and improve the interpretation. For instance, based on event locations and their source mechanisms, we can estimate discrete fracture network, compute stimulated rock volume, invert for local stress field or estimate pore pressure perturbations needed to activate faults (e.g., Zoback, 2007; Warpinski et al., 2013; Neuhaus et al., 2014). These additional parameters lead to better understanding and optimization of the reservoir stimulation resulting in an increased reservoir permeability and hydrocarbon production.

Each step in microseismic data processing carries some uncertainty and every uncertainty may affect data interpretation (e.g., Zimmer, 2011). For example, uncertainty in an event location negatively affects the source mechanism inversion and may project into a false fault plane or spurious non-shear components may be obtained. Not every inverted source mechanism has quality good enough to be used for further interpretation or, at least, should be treated with higher uncertainty.

The source mechanisms of microseismic events are usually inverted from the amplitudes and phases of the first arrivals of P- and S-waves (e.g. Jechumtalová and Eisner, 2008), alternatively from S/P amplitude ratios (Foulger et al., 2014). The inversion of fullwaveforms is not as common as in earthquake seismology because the reservoir models generally do not allow modeling the full waveforms at high frequencies - in microseismic monitoring, the signal strength usually peaks at frequencies in the band 20$40 \mathrm{~Hz}$ for surface monitoring and typically waveforms with peak frequencies $100 \mathrm{~Hz}$ and higher are used in borehole monitoring (Duncan and Eisner, 2010). Each source mechanism of a point source is mathematically fully described with a full moment tensor. It carries information about the orientation of the fault plane, sense of slip on the fault and the mode of the fracture. Studying shear and non-shear components is crucial 
for distinguishing pure-shear event from (partly) nonshear event indicating fracture opening or closing.

There are many sources of uncertainties influencing a source mechanism inversion, such as the data noise (e.g. Stanek et al., 2014; Mustać and Tkalčić, 2016), uncertainty in location and velocity model (e.g. Š́lený, 2009; Yagi and Fukahata, 2011), uncertainty in attenuation model, receiver array geometry, etc. The receiver array geometry is usually the only thing we can control. Therefore, we focused our study on the evaluation of this effect.

In this study, we compute the theoretical stability of source mechanisms for three examples of receiver arrays currently used in microseismic monitoring. Specifically, we compute a 3D distribution of condition numbers highlighting zones with good and bad quality of inverted full moment tensors representing source mechanisms. Although the condition numbers do not depend on the data, the condition numbers say how much the source mechanism parameters are stable and sensitive to a small change in the input: in our case amplitude, velocity model, event location, etc. We have chosen condition numbers as one of the commonly used and robust measures of inversion stability, but other parameters might be used to evaluate source mechanism quality as well, e.g., error ellipsoid (Zahradník and Custodio, 2012). However, none of the other measures represents perfectly the uncertainty and each of the measures of the source mechanism stability has both drawbacks and advantages.

We mimic three different types of arrays used to monitor reservoir stimulation that were used in publications interpreting source mechanisms of microseismic events. We test a dense surface array (e.g., Anikiev et al., 2014), dual borehole array (Jechumtálová et al., 2016) and receiver arrays in many shallow boreholes (Mahrooqi et al., 2013).

The goal of this study is not to criticize or praise any type of the arrays. Every array is designed for a specific purpose, and not necessarily for estimating the source mechanisms, and is limited by different rock types and surface infrastructures. Also, every project has a limited budget. Our goal is to quickly predict the quality of source mechanisms and possibly avoid an erroneous interpretation of microseismic results. Another goal is to provide a tool for an improvement of array design and selecting appropriate installations to optimize the cost of microseismic monitoring.

\section{METHODOLOGY}

For each selected receiver array, we set an area of interest (the stimulated area) where we test the theoretical assessment of the full-moment-tensor resolvability. Surface and near-surface arrays have all the expected events below the arrays, unlike the deeper borehole arrays which are intentionally installed closer to the stimulated part of the reservoir and events occur at similar depths as the depths of receivers.

In the area of interest, we define a regular grid of potential microseismic event locations for which the stability of source mechanism inversion is computed. We assume that all receivers of the arrays contribute with the signal ( $\mathrm{P}$ - or $\mathrm{P}$ - and $\mathrm{S}$-wave amplitudes). Hence, our study presents the best-case scenario for the strongest events, i.e. events with sufficient signalto-noise ratio at the monitoring arrays as discussed later.

We compute synthetic first arrival amplitudes (either positive or negative values) of direct P- and Swave for the receivers from each point of the grid of potential microseismic event locations. For simplicity, we assume a homogeneous isotropic medium and a point source; however, we realize that more complex media would affect the source mechanism inversion. The point source is a good approximation for microseismic events which are smaller than magnitude 0 . The receiver array configuration has a dominant effect because sufficient receiver coverage overcomes uncertainties in the velocity model (Š́lený, 2009). We neglect the noise effect and assume that we are able to correctly pick the right arrival amplitudes at every receiver, i.e., that we have a sufficient signalto-noise ratio at every receiver. The latter one is quite a strong assumption for all the tested arrays, but this study evaluates ability to correctly invert for the source mechanism of the well recorded microseismic events. This means that the predicted stability of the inversion is true only for the events strong enough to be recorded on all receivers of the tested monitoring array. Generally, the downhole monitoring arrays achieve high signal-to-noise ratio even for weaker events, it is not true for surface monitoring arrays. In such sense the condition number represents an ideal situation and real data may only degrade such assumption. We remark that downhole monitoring array tests assume the inversion of S-waves in an isotropic velocity model, which may not be a suitable approximation in anisotropic media (Grechka, 2015).

We compute Green's function matrix $\boldsymbol{G}$ for the full moment tensor $\boldsymbol{M}$ inversion of the first P-wave arrival amplitudes (either positive or negative values see Eisner et al., 2010 with example of real data picking strategy) for the surface vertical component array and shallow boreholes receivers with only vertical components array, and another matrix of both the first P- and first S-wave arrival amplitudes modeled for the dual borehole array. The observed amplitudes of direct $\mathrm{P}$ - and S-wave displacement $\boldsymbol{d}$ can be related with $\boldsymbol{G}$ and $\boldsymbol{M}$ by equation

$d=G * M$.

An example of matrixes $\boldsymbol{d}$ and $\boldsymbol{G}$ for one threecomponent station and both $\mathrm{P}$ - and S-waves looks like this

$$
\boldsymbol{d}=\left[\begin{array}{l}
A_{P N} \\
A_{P E} \\
A_{P Z} \\
S_{P N} \\
S_{P E} \\
S_{P Z}
\end{array}\right], \boldsymbol{G}=\left[\begin{array}{l}
G_{P N}(1) G_{P N}(2) \ldots G_{P N}(6) \\
G_{P E}(1) G_{P E}(2) \ldots G_{P E}(6) \\
G_{P Z}(1) G_{P Z}(2) \ldots G_{P Z}(6) \\
G_{S N}(1) G_{S N}(2) \ldots G_{S N}(6) \\
G_{S E}(1) G_{S E}(2) \ldots G_{S E}(6) \\
G_{S Z}(1) G_{S Z}(2) \ldots G_{S Z}(6)
\end{array}\right]
$$


Here $A_{P N}$ is an amplitude of P-wave displacement measured on north component; $G_{P N}(1)$ is the P-wave Green's function's derivative for a farfield ray approximation of north component amplitude due to the first component of moment tensor $\boldsymbol{M}$; numbers (1)...(6) denote all six independent components of $\boldsymbol{M}$. Time variation of $G * M$ in a far-field ray approximation is delta function. That is why at every station each component is represented by just a single time sample (at the first arrival P- or Swave).

The least-square moment tensor inversion of linear equation (1) is

$$
M=\left(G^{T} G\right)^{-1} G^{T} d
$$

The condition number $C N$ is defined as a square root of a ratio between the maximum and the minimum eigenvalue of the symmetric inversion matrix $\boldsymbol{G}^{T} \boldsymbol{G}$ in equation (2):

$$
N=\sqrt{\frac{\max \left[\operatorname{eig}\left(G^{T} G\right)\right]}{\min \left[\operatorname{eig}\left(G^{T} G\right)\right]}},
$$

A well-conditioned inversion has a low condition number and its result is stable, whereas ill-conditioned inversion has a high condition number and the resulting source mechanism is very sensitive to any small change in the input. While low condition number does not guarantee correctly inverted mechanism (due to uncertain velocity and attenuation models, incorrect picks, etc.), the high condition number means highly unstable and probably incorrect source mechanism even if the event is well recorded with a high signal-to-noise ratio. Note that determination of the condition number does not require any information about the data and is only dependent on matrix $\boldsymbol{G}$, hence on source-station configuration and the assumed velocity model.

The specification of a condition number threshold between well- and ill-conditioned inversion is not clear and also depends on the inversion parametrization. Dufumier and Rivera (1997), using the same condition number definition as we do in this study, suggest a condition number magnitude providing reliable results in order of first tens, whereas, Nolen-Hoeksema and Ruff (2001) or Vera Rodrigues et al. (2011), using condition number definition without the square root, get results with sufficient quality using the condition numbers as large as 500. Therefore, because we are interested just in relative resolvability measure, we tentatively assume that a reasonable upper limit for a reliable source mechanism inversion is 20 and we show the condition numbers on a scale from 1 to 20 . Our results are 3D grids of condition numbers showing zones where the full moment tensors describing source mechanisms are expected to be stable and reliable for an interpretation.

\section{STABILITY FOR THREE TYPES OF MICROSEISMIC MONITORING ARRAYS}

The first tested monitoring array is a surface starlike array with 800 receivers in 8 arms around the wellhead, similar to the array of Anikiev et al. (2014). Each arm is $2.5 \mathrm{~km}$ long and the whole array is covering approximately $25 \mathrm{~km}^{2}$. The area of interest where we expect induced events is around the stimulated lateral parts of the four hypothetical treatment wells at the depth $2 \mathrm{~km}$. Inverting P-wave amplitudes from all the receivers on vertical components, we get the lowest condition numbers around 5 (the most stable results) for the locations below the middle of the array (Figure 1). The quality of the inverted source mechanisms decreases to the edges of the star and with the depth. The events located deeper than $2300 \mathrm{~m}$, i.e., approximately the length of the arm, have source mechanisms inverted very poorly.

Based on our results, the inverted source mechanisms of real events presented by Anikiev et al. (2014) or Staněk and Eisner (2013) are stable and could be used for further interpretations. This statement neglects the noise effect, but Staněk et al. (2014) showed that a source mechanism inversion using the method of equation (2) represents the stacking which suppresses the noise and results in reliable source mechanisms even for weak events. The results of Anikiev et al. (2014) are consistent with this conclusion, as they achieve high stability of the inverted strikes even for weak events with moment magnitudes down to -1.7 .

The second tested array configuration is a dual borehole monitoring array with 11 receivers in each well, similar to Jechumtálová et al. (2016). The vertical wells are drilled $500 \mathrm{~m}$ from each other and the receivers are located at depths ranging from 1800 to $1920 \mathrm{~m}$. In this case, we use as input both P- and Swave amplitudes, because they are commonly used for borehole receivers. The most stable source mechanisms for this array are estimated between the two wells, but not along the plane connecting them as shown in Figure 2. The color scale is the same as in Figure 1. The best possible inversion with $\mathrm{CN}=7.7$ is achieved for an event located at the depth of the center receivers, and with the angle $45^{\circ}$ between the event location and the plane containing both wells. The unstable results depicted by high condition numbers are for events located too far away from the wells, and close to the plane containing both wells and in the vicinity of each well. These results are consistent with Vavryčuk (2007), who showed that the full moment tensor is not constrained along the plane containing both monitoring wells. This problem could be practically solved using arrays in slightly deviated wells (Vera Rodrigues et al., 2011) which improves focal sphere coverage in the problematic direction, or by using a constrained inversion (Jechumtálová and Š́lený, 2005). Luckily, events located by Jechumtálová et al. (2016) seems to be in a zone (cut by cross-sections in Fig. 2) where we get relatively 
low condition numbers, indicating stable inversion of the observed source mechanisms.

The inversion from the surface array seems stable for most stimulation area because of the higher number of receivers and better focal sphere coverage. Comparing to (near-)surface arrays, the key advantage of borehole receiver arrays is the short distance from the induced events, the resulting availability of Swave amplitudes and the detectability of low magnitude events.

The third tested array consists of 13 shallow boreholes arrays with 104 receivers in total, as illustrated in Figure 3. A similar array was used by Mahrooqi et al. (2013) to study source mechanisms in Oman. Although this array is designed to monitor a stimulation in a very shallow reservoir, we test the source mechanism inversion from only P-wave amplitudes, because $\mathrm{S}$-waves are very complex in shallow horizontal propagation. For such receiver geometry, we get a very good stability of the source mechanisms inverted below the center of the (multi-)borehole array at the depths exceeding $300 \mathrm{~m}$ and optimally around $700 \mathrm{~m}$, as shown by the red area in Figure 3 with minimum $\mathrm{CN}=2.7$. At this depth, comparable to the offsets of the monitoring wells, we have high stability and good focal sphere receiver coverage, similarly as for the surface array. Unfortunately, the real stimulated reservoir is shallower and the monitoring was designed to detect events at depths shallower than $500 \mathrm{~m}$. At these depths, the focal sphere coverage is worse. For some locations, we get extremely high condition numbers because most of energy comes from the receivers, which are close to the horizontal plane of the inverted source mechanisms. Horizontal rays do not constrain the vertical component of moment tensor, as it is well known in global seismology (Lay and Wallace, 1995). This situation is similar to the borehole array.

A better stability of the source mechanism inversion could be achieved by using S-wave amplitudes on the shallow borehole array. However, using all P-wave arrivals for all the receivers in the shallow reservoir is likely to be too optimistic. Mahrooqi et al. (2013) observed useful signal only on a few neighboring wells (usually two). In such a case, the source mechanism stability is very similar to our second tested array - the dual monitoring borehole array. Using both $\mathrm{P}$ - and S-wave arrivals for two near boreholes may solve the problem with events located in the depths of receivers close to the wells. However, the stability of the inversion from two boreholes only has limitations and the best quality of inversion is achieved between the wells, $45^{\circ}$ from the plane containing both wells. Thus, a reliable inversion requires the monitoring boreholes to be drilled closer to each other than in the case presented by Mahrooqi et al. (2013). A possible improvement for such a shallow reservoir could be adding (near-) surface stations, but these stations would be probably noisy. Another option would be extending the monitoring wells to a greater depth and making the monitoring arrays longer to improve the focal sphere receiver coverage.

\section{DISCUSSION AND CONCLUSIONS}

We computed 3D distributions of condition numbers for three commonly used types of receiver arrays in microseismic monitoring. Note that this methodology is not dependent on the actual acquired data but is derived from synthetic medium model (equation 1 and 2), we assume homogenous isotropic medium for simplicity. Each of the three tested arrays provides specific result, although there are some similarities too. The surface array (first tested array) has low condition numbers (i.e. good invertability) similarly to the (multi-)array of shallow boreholes (third tested array), at depths comparable with the distance between the center of the array and the most distant receiver. High condition numbers are found around and between the wells for both, dual-borehole array (second tested array) and array of shallow boreholes, indicating low stability of inverted full moment tensor source mechanisms.

In this study, we assumed the best possible scenario: all amplitudes are contributing on all stations, i.e., P-wave for surface and shallow borehole array and P- and S-waves for dual borehole array. Considering low magnitude microseismic events and a realistic signal-to-noise ratio, we might not be able to correctly pick the amplitudes at each receiver and this would deteriorate real resolvability of moment tensor. For example, Mahrooqi et al. (2013) did not have sufficient signal-to-noise ratio to reliably pick the amplitudes on all the receivers and used data from only limited number of wells. The surface monitoring array of Anikiev et al. (2014) and the dual borehole monitoring array of Jechumtalová et al. (2016) detected events which had sufficient signal on the most of the receivers.

Our study clearly shows that there are places where some arrays do not allow to invert stable full moment tensor even in the best scenario situation. The ability of using all receivers is not a major problem for a dense surface array, where are hundreds of receivers, but it is critical for borehole arrays. There it is necessary to record reliable $\mathrm{P}$ - and $\mathrm{S}$-wave amplitudes on as many receivers as possible to achieve as large as possible solid angle (an angle given by the aperture of receiver array, as viewed from the source location) resulting in a stable source mechanism inversion. We remark also that the velocity model affects more severely the borehole arrays, where guided and head waves may interfere with reflected and direct arrival waves, and anisotropy affects severely S-waves.

The source mechanism inversion can be made more stable by adding more optimally placed stations, using S-wave amplitudes for surface or near surface receivers or adding another input parameter constraining the mechanism such as $\mathrm{SH} / \mathrm{P}$ or $\mathrm{SV} / \mathrm{P}$ amplitude ratios.

The key factor for the source mechanisms inversion is the solid angle or in other words the focal sphere coverage. For weak events in records with low signal-to-noise ratio recorded only by the downhole arrays, it is important to record arriving waves in the largest possible solid angle (e.g., Eaton and 
Forouhideh, 2011). For strong events, a large solid angle is achievable with (near-)surface arrays.

The presented results neglect the effects of velocity and attenuation model. They show where the moment tensor inversions are (un-)stable due to a receiver configuration. Therefore, the results in this study in some sense represent the most optimistic estimate we can achieve.

\section{ACKNOWLEDGMENTS}

We would like to thank The Petroleum Institute (grant n.14501), Charles University grant SVV 260447/2017 and SEG/Chevron for their support. This work was carried out thanks to the support of the long-term conceptual development research organization RVO: 67985891. We are really grateful to Prof. Jiří Zahradník from Charles University in Prague, Faculty of Mathematics and Physics, Dept. of Geophysics for his helpful comments and discussion.

\section{REFERENCES}

Anikiev, D., Valenta, J., Staněk, F. and Eisner, L.: 2014, Joint location and source mechanism inversion of microseismic events: benchmarking on seismicity induced by hydraulic fracturing. Geophysical Journal International, 198, 1, 249-258.

DOI: $10.1093 / \mathrm{gji} / \mathrm{ggu} 126$

Dufumier, H. and Rivera, L.: 1997, On the resolution of the isotropic component in moment tensor inversion. Geophysical Journal International, 131, 3, 595-606. DOI: 10.1111/j.1365-246X.1997.tb06601.x

Duncan, P. and Eisner, L.: 2010, Reservoir characterization using surface microseismic monitoring. Geophysics, 75, 5, 75A139-75A146. DOI:10.1190/1.3467760

Eaton, D.W. and Forouhideh, F.: 2011, Solid angles and the impact of receiver-array geometry on microseismic moment-tensor inversion. Geophysics, 76, 6, WC75WC83. DOI: 10.1190/GEO2011-0077.1

Eisner, L., Williams-Stroud, S., Hill, A., Duncan, P. and Thornton, M.: 2010, Beyond the dots in the box: Microseismicity-constrained fracture models for reservoir simulation. The Leading Edge, 29, 3, 326333. DOI: $10.1190 / 1.3353730$

Foulger, G.R., Julian, B.R., Hill, D.P., Pitt, A.M., Malin, P.E. and Shalev, E.: 2004, Non double-couple microearthquakes at Long Valley caldera, California, provide evidence for hydraulic fracturing. Journal of Volcanology and Geothermal Res., 132, 45-71. DOI: 10.1016/S0377-0273(03)00420-7

Grechka, V.: 2015, On the feasibility of inversion of singlewell microseismic data for full moment tensor. Geophysics, 80, 4, KS41-KS49.

DOI: 10.1190/geo2014-0471.1

Jechumtálová, Z., Chu, F., Rong, J., Procházka, J. and Eisner, L.: 2016, Downhole Microseismic Data Processing - Consistency of Locations, Source Mechanisms and Stress State. 78th EAGE Conference \& Exhibition, Extended Abstract. DOI: $10.3997 / 2214-4609.201600720$

Jechumtálová, Z. and Eisner, L.: 2008, Seismic source mechanism inversion from a linear array of receivers reveals non-double-couple seismic events induced by hydraulic fracturing in sedimentary formation. Tectonophysics, 460, 1-4, 124-133. DOI: $10.1016 /$ j.tecto.2008.07.011

Jechumtálová, Z. and Š́lený, J.: 2005, Amplitude ratios for complete moment tensor retrieval. Geophysical
Research Letters, 32, 22, L22303.

DOI: 10.1029/2005GL023967

Lay, T., and Wallace, T.C.: 1995, Modern Global Seismology, Academic Press, San Diego, 313-356.

Mahrooqi, S., Busaidi, S., Ismaili, I., Clow, F., Urbancic, T., Baig, A. and Kassam, A.: 2013, Beyond the dots Microseismic monitoring of a fractured reservoir during steam injection in Oman. 75th EAGE Conference \& Exhibition, Extended Abstract. DOI: $10.3997 / 2214-4609.20130014$

Mustać, M. and Tkalčić, H.: 2016, Point source moment tensor inversion through a Bayesian hierarchical model. Geophysical Journal International, 204, 1, 311-323. DOI:10.1093/gji/ggv458, 2016

Neuhaus, C.W., Ellison, M., Telker, C. and Blair, K.: 2014, Drainage estimation and proppant placement evaluation frommicroseismic data. SPE/EAGE European Unconventional Resources Conference and Exhibition, Extended abstract. DOI: $10.2118 / 167685-M S$

Nolen-Hoeksema, R. and Ruff, L.J.: 2001, Moment tensor inversion of microseisms from the B-sand propped hydrofracture, M-site, Colorado. Tectonophysics, 336, 1, 163-181. DOI: 10.1016/S0040-1951(01)00100-7

Š́lený, J.: 2009, Resolution of non-double-couple mechanisms: Simulation of hypocenter mislocation and velocity structure mismodelling. Bulletin of the Seismological Society of America, 99, 4, 2265-2272. DOI: $10.1785 / 0120080335$

Staněk, F. and Eisner, L.: 2013, New model explaining inverted source mechanisms of microseismic events induced by hydraulic fracturing, SEG Technical Program. Expanded Abstracts, 2201-2205. DOI: $10.1190 /$ segam2013-0554.1

Staněk, F., Eisner, L. and Moser, T.J.: 2014, Stability of source mechanisms inverted from P-wave amplitude microseismic monitoring data acquired at the surface. Geophysical Prospecting, 62, 3, 475-490. DOI: 10.1111/1365-2478.12107

Vavryčuk, V.: 2007, On the retrieval of moment tensors from borehole data. Geophysical Prospecting, 55, 3, 381-391. DOI: 10.1111/j.1365-2478.2007.00624.x

Vera Rodrigues, I., Gu, Y.J. and Sacchi, M.D.: 2011, Resolution of seismic-moment tensor inversions from a single array of receivers. Bulletin of the Seismological Society of America, 101, 6, 2634-2642. DOI: $10.1785 / 0120110016$

Warpinski, N.R., Mayerhofer, M.J., Agarwal, K. and Du, J.: 2013, Hydraulic fracture geomechanics and microseismic source mechanisms. SPE Journal, 18, 4, Society of Petroleum Engineers. DOI: $10.2118 / 158935-\mathrm{PA}$

Yagi, Y. and Fukahata, Y.: 2011, Introduction of uncertainty of Green's function into waveform inversion for seismic source processes. Geophysical Journal International, 186, 711-720. DOI: 10.1111/j.1365-246X.2011.05043.x

Zahradník, J. and Custódio, S.: 2012, Moment tensor resolvability: Application to Southwest Iberia. Bulletin of the Seismological Society of America, 102, 3, 1235-1254. DOI: 10.1785/0120110216

Zimmer, U.: 2011, Calculating sitimulated reservoir volume (SRV) with consideration of uncertainties in microseismic-event locations. Canadian Unconventional Resources Conference. SPE Journal, SPE148610-MS, Society of Petroleum Engineers. DOI: $10.2118 / 148610-M S$

Zoback, M.D.: 2007, Reservoir geomechanics. Cambridge University Press, 340-377. 

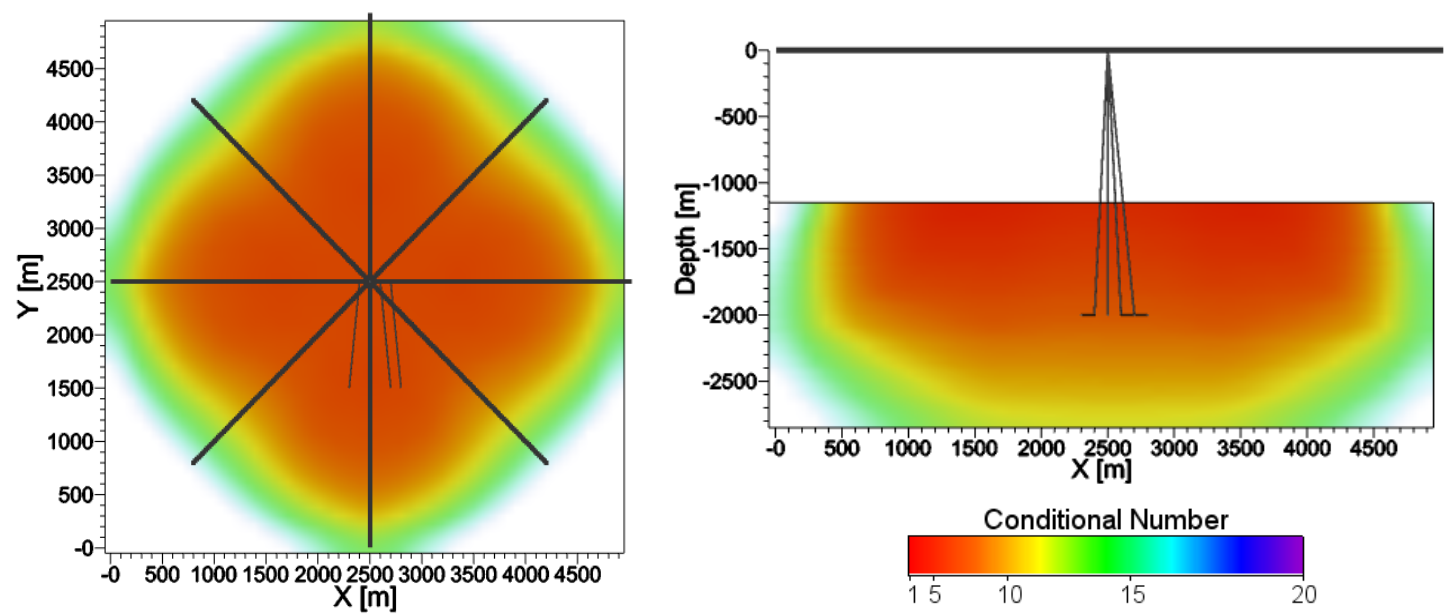

Fig. 1 Horizontal and vertical cross-section slices of condition numbers computed for a dense surface star-like array (black lines). The hypothetical horizontal treatment wells are shown as thin black lines in the horizontal (at the depth $2 \mathrm{~km}$ ) and the vertical (at $\mathrm{Y}=2500$ $\mathrm{m})$ cross-sections. The quality of the inverted source mechanisms decreases for higher condition numbers, from red to blue. The values above 20 are shown as white color.
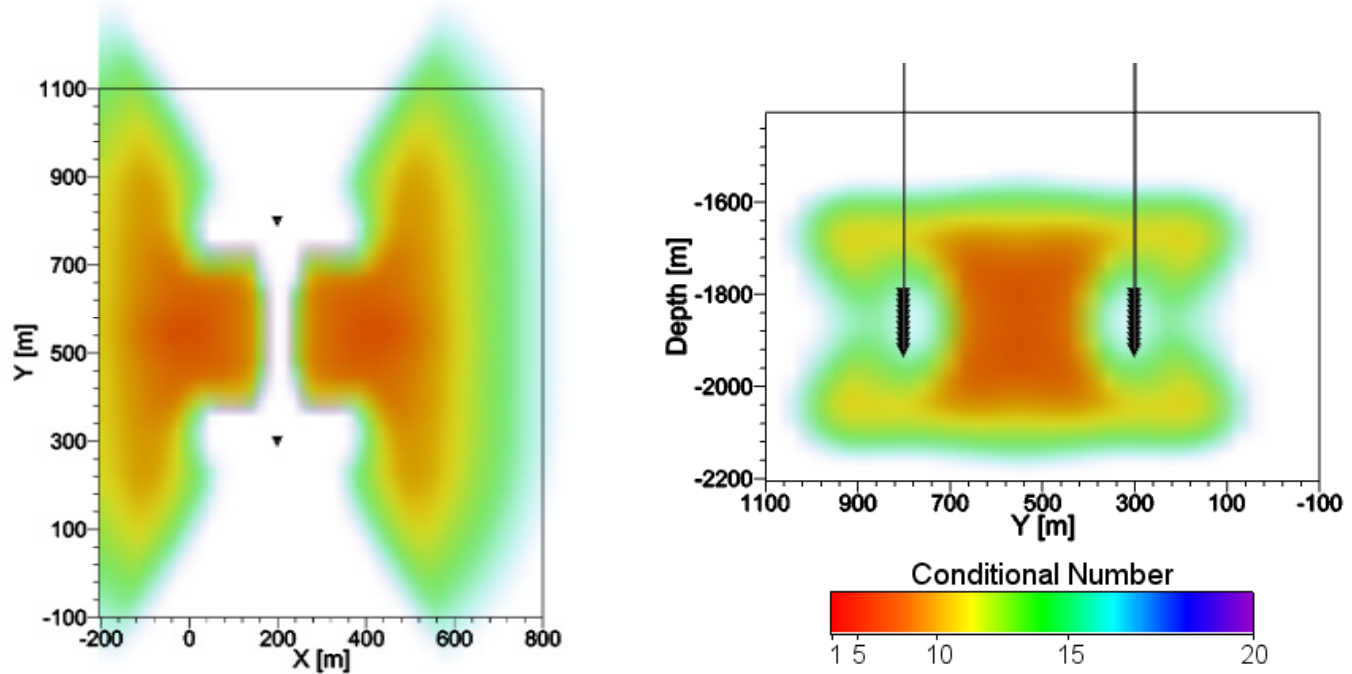

Fig. 2 Horizontal and vertical cross-sections of condition numbers computed for a dual borehole array. The wells are shown as thin black lines and the receivers of the monitoring array are shown as black upside-down triangles. The condition numbers are shown at the horizontal (at the depth $1820 \mathrm{~m}$ ) and the vertical (at $\mathrm{X}=370 \mathrm{~m}$ ) cross-section slices.
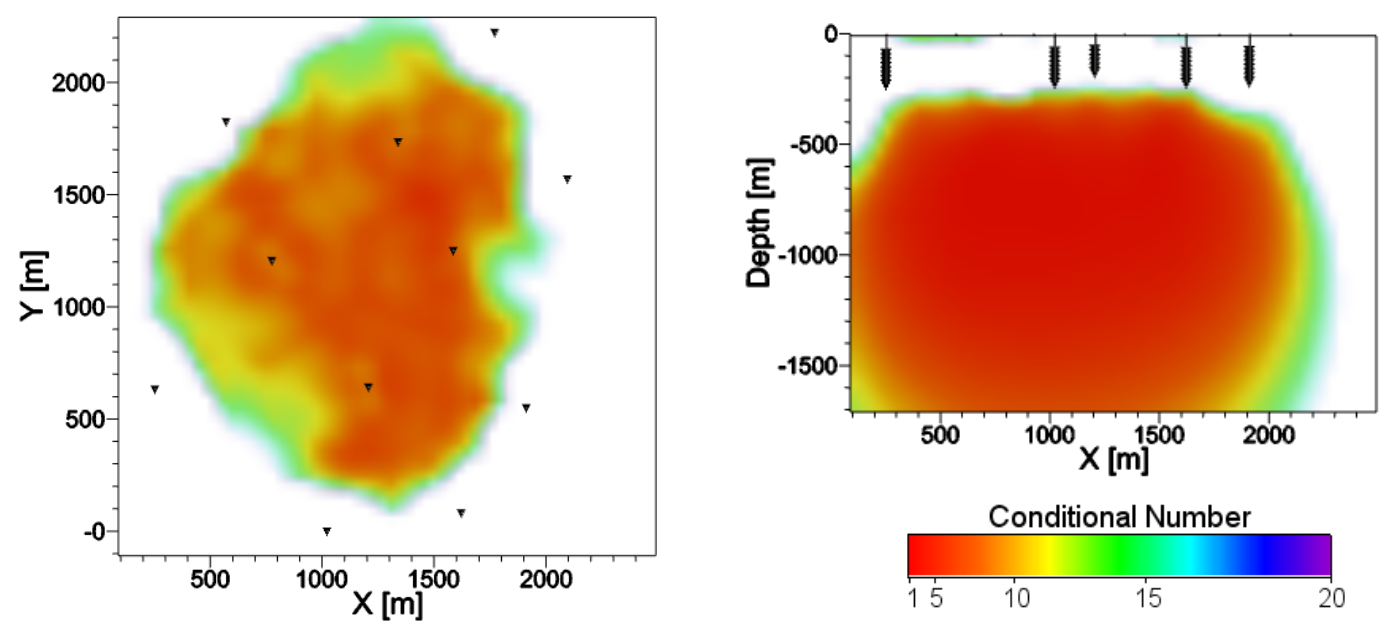

Fig. 3 Horizontal and vertical cross-sections of condition numbers computed for receiver array installed in shallow boreholes, represented by the black upside-down triangles projected to the plane. The condition numbers are shown at the horizontal (at the depth $330 \mathrm{~m}$ ) and the vertical cross-sections $(\mathrm{Y}=1100 \mathrm{~m})$. 